\title{
Efeito da reabilitação vestibular em paciente pós traumatismo cranioencefálico (TCE): relato de caso
}

\author{
Effects of vestibular rehabilitation on patient after TBI: case report \\ Maria da Glória Canto de Sousa ${ }^{1}$, Cristina Ganança², Eduardo Pondé de Sena ${ }^{3}$ \\ ${ }^{1}$ Fonoaudióloga. Doutoranda do Programa de Pós-graduação Processos Interativos dos \\ Órgãos e Sistemas, ICS - UFBA Professora Assistente de Fonoaudiologia. UNEB \\ ${ }^{2}$ Professora Adjunto de Fonoaudiologia. Escola Paulista de Medicina. UNIFESP \\ ${ }^{3}$ Professor Associado. Departamento de Biorregulação. Programa de Pós-graduação \\ Processos Interativos dos Órgãos e Sistemas, ICS - UFBA
}

\begin{abstract}
Resumo
Introdução: O controle postural é uma das mais complexas funções sensoriomotoras do corpo, uma vez que a perfeita harmonia na execução e compreensão das informações vestibular, visual e proprioceptiva é integrada pelo sistema nervoso central (SNC). A perturbação na integração desses sistemas determina prejuízos motores, sensitivos, e/ou cognitivos os quais comprometem a mobilidade do indivíduo. O Traumatismo Crânio Encefálico (TCE) é um acontecimento que pode levar o paciente à morte ou à incapacidade crônica, o que invariavelmente desestabiliza seu meio familiar e interfere em sua vida social e/ou profissional. O tratamento do paciente sequelado por trauma crânio encefálico promove, de forma significativa, a recuperação do equilíbrio, sobretudo quando se trabalham os aspectos relacionados ao reflexo vestíbulo-ocular. A reabilitação vestibular é um recurso terapêutico indicado no tratamento de pacientes com distúrbios do equilíbrio corporal; sua eficácia está baseada nos mecanismos relacionados à plasticidade neuronal do SNC. Objetivo: descrever a contribuição da terapia labiríntica baseada na habituação no paciente acometido por trauma cranioencefálico com sequelas cerebelar e labiríntica. Metodologia: o presente estudo consiste no relato do caso clínico de um paciente de cinquenta e cinco anos de idade, atendido e acompanhado no setor de Otoneurologia da Clínica Escola de Fonoaudiologia da UNEB, no período de 2008 a 2013. Os procedimentos utilizados seguiram o protocolo de atendimento ao paciente acometido com tontura do referido setor a saber: explanação acerca do funcionamento do programa; exposição teórica sobre anatomofisiopatologia do sistema vestibular; anamnese fonoaudiológica direcionada ao sistema auditivo e vestibular; aplicação do DHI (Dizziness Handicap Inventory) pré e pós-tratamento; utilização de protocolo de relaxamento de cintura escapular; realização de estimulação optocinética; realização dos exercícios dos protocolos de Cawthorne e Cooksey,e, do Zee e da Associação Italiana de Otologia; utilização de jogos com realidade virtual - Knect Xbox. Após a utilização de todos os procedimentos preconizados para o caso em questão, foi constatada a melhora do equilíbrio corporal (estático e dinâmico). Conclusão: Com base nos resultados obtidos, pode-se afirmar que a opção de utilizar protocolos diversos que venham a compor um programa de reabilitação do sistema labiríntico, principalmente, nos pacientes portadores de vestibulopatias mistas, como o caso ora exposto, favoreceu na recuperação das atividades básicas do dia a dia desse paciente, sobretudo quando da associação à estratégia terapêutica de jogos com realidade virtual. Assim, foi constatado que a associação desse procedimento aos protocolos tradicionais certamente propiciou maior possibilidade de atingir o fenômeno da habituação e adesão ao tratamento, possibilitando a recuperação do equilíbrio corporal, conferindo autonomia ao paciente.

Palavras-chave: Reabilitação labiríntica. Traumatismo Crânio Encefálico. Vertigem. Equilíbrio Postural.
\end{abstract}

Abstract

Background: Postural control is one of the most complex sensory motor functions of the human body, since the perfect harmony in the execution and understanding of vestibular, visual and proprioceptive information is integrated by the central nervous system (CNS). A disturbance in the integration of such systems determines motor, sensitive and/or cognitive damage that compromises the mobility of the individual. Traumatic brain injury (TBI) is an event that may lead to a patient's death or chronic incapacity, which invariably affects their family environment and interferes in their social/professional lives. The treatment of such patients promotes a significant recovery of their balance, especially when aspects related to the vestibule-ocular reflex are worked with. Vestibular rehabilitation is a therapeutic tool indicated for the treatment of patients with physical balance impairment; its efficiency is based on the mechanisms related to the neuronal plasticity of the CNS. Objective: To describe the contribution of labyrinthine therapy based on the habituation in a patient suffering from brain injury trauma with labyrinthine and cerebellar damage. Methodology: Case study of a 55 year-old male patient treated at the Otoneurology Department of Clínica de Fonoaudologia at UNEB between 2008 and 2013. The procedure used followed the care protocol of the referred department for patients suffering from dizziness: information on how the program works, theoretical explanation on anatomophysiopathology of the vestibular system, phonoaudiological anamnesis directed to the auditory and vestibular systems, application of DH (Dizziness Handicap Inventory) pre and post treatment, use of the relaxation protocol of the pectoral girdle, nystagmus stimulation, use of Cawthorne, Cooksey, zee and the Italian Association of Otology, use of virtual reality games such as Knect Xbox. Results: After the use of all the recommended procedures for the case in question, an improvement of the static and dynamic physical balance of the patient was observed. Conclusion: Based on the results obtained, it is possible to affirm that the choice for assorted protocols that may compose a labyrinthine system rehabilitation program, especially in patients with mixed vestibulopathy, as in the present case study, has shown to be useful for the recuperation of the basic daily life activities performed by the patient, mainly after the strategic therapy with virtual games. That way, it has been found that its association to the traditional protocols has lead to a higher possibility of achieving the patient's habituation and adherence to the treatment, bringing back the patient's balance as well as his autonomy. Keywords: Labyrinthine rehabilitation. Traumatic Brain Injury (TBI). Dizziness. Physical balance.

Correspondência / Correspondence: Maria da Glória Canto de Sousa. Programa de Pós-graduação Processos Interativos dos órgãos e sistemas. Av. Reitor Miguel Calmon, S/N, Vale do Canela. CEP: 40110-100. Salvador -BA Fone: (71) 3283-8959 E-mail:gloriacanto@yahoo.com.br 


\section{INTRODUÇÃO}

O controle postural é uma das mais complexas funções sensoriomotoras do corpo, uma vez que é a perfeita harmonia na execução e compreensão das informações vestibular, visual e proprioceptiva é integrada pelo sistema nervoso central (SNC). Qualquer intempérie na integração desses sistemas determina prejuízos motores, sensitivos e cognitivos os quais comprometem a mobilidade do indivíduo. Lesões no trato córtex cerebral são exemplos de causa para desintegração entre os sistemas vestibular, proprioceptivo e visual, expondo o indivíduo aos sintomas de vertigem.

O Traumatismo Crânio Encefálico (TCE) é um acontecimento que pode levar o paciente à morte ou à incapacidade crônica, o que invariavelmente desestabiliza seu meio familiar e interfere em sua vida social e/ou profissional (FONSECA, 2013). Quando não provoca comorbidades, a esperança média de vida é idêntica à de um indivíduo saudável com a mesma idade e sexo (BROWN et al., 2011).

Díaz (2011), em referência a Masini (2010), cita que, no Brasil, existem poucos estudos epidemiológicos de TCE, estimando "uma incidência anual de 341 casos por 100.000 habitantes com uma taxa de internação de 0,5 por 1.000 habitantes para a cidade de Brasília, no ano de 1991, incluindo apenas os residentes dessa cidade".

Segundo Masel e Dewitt (2010), o TCE não é apenas um evento, mas sim um processo que pode ser considerado como uma doença crônica; trata-se de um problema de saúde pública o qual gera um importante impacto econômico e social, tamanhas são suas consequências com grave acometimento da funcionalidade do indivíduo em diferentes áreas.

A tontura e o desequilíbrio são sequelas que comumente acompanham o paciente pós TCE, sobretudo quando decorre lesão cerebelar. Transtornos do sistema vestibular implicam vertigens contínuas e intermitentes, além da redução do equilíbrio corporal, incluindo o aumento do risco de quedas (HOFFER; VIIRRE, 2012). As alterações do equilíbrio corporal podem ser secundárias a comprometimentos na interação entre os sistemas sensorial e motor envolvendo o vestíbulo, o qual é responsável pela posição e pelo movimento da cabeça; a propriocepção, relacionada à postura e à movimentação do corpo; e visual, que tem a ver com as relações espaciais. Tais sistemas permitem que o corpo mantenha-se parado, de modo estável, ou em movimento, mas de maneira harmônica (GANANÇA et al., 2008). Na ausência de um sistema vestibular funcional, o SNC apresentará dificuldades para integrar adequadamente as informações provenientes dos sistemas visuais e proprioceptivos (BITTAR; BARROS, 2011).

$O$ paciente com queixa de tontura tende a relatar dificuldade de concentração mental, além de perda de memória e fadiga. Esse estado leva-o a uma insegurança física gerada pela tontura e pelo desequilíbrio, o que pode conduzir à irritabilidade, perda da autoconfiança, medo de sair sozinho, ansiedade, depressão ou pânico (CABRAL et al., 2009).

$\mathrm{Na}$ intenção de reduzir os sintomas desagradáveis, os sujeitos restringem suas atividades profissionais, sociais e domésticas, piorando sua qualidade de vida (QV) (GANANÇA; CAOVILLA, 1998).

No quadro de traumatismo craniano leve, como resultado, tem-se um comprometimento do reflexo vestíbulo ocular (RVO) o qual pode apresentar deficits no equilíbrio corporal por períodos prolongados de tempo (RECKARD et al., 2013).

O paciente com sequelas após um TCE pode ter comprometimentos em outras partes do SNC, o que pode vir a trazer complicações quanto a sua recuperação. Assim, o traumatismo pode [...] restringir os movimentos; trazer problemas musculoesqueléticos e neuromusculares os quais afetam a estabilidade quando o paciente necessitará movimentar-se para alcançar a compensação do SNC; além de haver a possibilidade de lesões nos sistemas visual e somatossensitivo, limitando a disponibilidade dessas sensações como alternativas para as informações vestibulares perdidas [...] (SHUMWAY-COOK, 2002).

Segundo os autores, o tratamento do paciente sequelado por trauma crânio encefálico promove a recuperação do equilíbrio o qual pode melhorar, de forma significativa, sobretudo quando se trabalham os aspectos relacionados ao reflexo vestíbulo ocular (HOFFER et al., 2004).

A partir das recomendações do Consenso sobre Vertigem, realizado pela Sociedade Brasileira de Otorrinolaringologia (2000), foi preconizada, como medida terapêutica, a realização de exercícios para reabilitação vestibular quando o paciente cursa com tontura. $O$ protocolo é baseado nas seguintes etapas: orientação ao paciente e sua família, esclarecendo o propósito do funcionamento básico do equilíbrio, de seus sintomas e do tratamento proposto; em seguida iniciar o treinamento da Reabilitação Vestibular, composto por movimentos repetidos de olhos, cabeça, tronco e exercícios de marcha; e ao final, é orientado sobre a necessidade de acompanhamento e monitoração, enfatizando-se as orientações para fins de acompanhar a evolução durante período de três meses (TAGUCHI, 2009; NEWMAN; JACOBSON; SPITZER, 1996).

A reabilitação vestibular é um recurso terapêutico indicado e aplicado no tratamento de pacientes com distúrbios do equilíbrio corporal; sua atuação está baseada nos mecanismos relacionados à plasticidade neuronal do SNC (GANANÇA ; GANANÇA, 2001; GANANÇA; CAOVILLA ; MUNHOZ, 1997). A reabilitação vestibular além de promover a estabilização visual durante os movimentos da cabeça, melhora a interação vestíbulo visual durante a movimentação cefálica e, ainda, amplia a estabilidade postural estática e dinâmica nas condições que produzem informações sensoriais conflitantes (GANANÇA ; GANANÇA, 2001). 
Bittar et al. (2007) reiteram o valor da reabilitação vestibular no tratamento das vestibulopatias reconhecendo-a como uma terapia auxiliar ou única; no entanto, seu prognóstico depende da etiologia da tontura.

Segundo Silva e Moreira (2000), a reabilitação vestibular passa pelo processo de habituação que se baseia nos estímulos repetitivos os quais levam à compensação vestibular central, um processo de adaptação em que ocorre a substituição das informações geradas no labirinto lesado por estímulos advindos dos sistemas visual, proprioceptivo, entre outros. Esse processo envolve a reaprendizagem sensório-motora realizada por estruturas integradas do SNC. Citando Shepard e Telian (1995), os autores acima fazem referência às lesões estáveis do SNC ou portadores de lesões mistas (centrais e periféricas) como sendo pacientes passíveis de tratamento, contudo seu prognóstico é reservado quando comparados aos portadores de disfunções periféricas estáveis. As lesões mistas dificultam o processo de compensação vestibular central. Missaoui e Thoumie (2009) reforçam a importância de os pacientes serem tratados em uma perspectiva multisensorial, ou seja, estimulando os sistemas visual, vestibular e proprioceptivo e que sejam de acordo com seu nível de deficiência, sendo a forma mais adequada para a redução do comprometimento do equilíbrio em pacientes com esse tipo de lesão.

Nas últimas décadas, a realidade virtual (RV) tem sido uma estratégia utilizada para o tratamento de pacientes com tontura. Tal método envolve tecnologias revolucionárias para o desenvolvimento de aplicações com o uso de dispositivos multissensoriais, com navegação em espaços tridimensionais, imergindo em um contexto em tempo real, que amplia os sentidos anteriormente ligados à simples visualização, audição e manipulação tridimensional para o tato, pressão e até olfato (BARILLI et al., 2013).

A realidade virtual permite recriar ao máximo a sensação de interação do indivíduo com o computador, no qual a percepção do ambiente é modificada por um estímulo sensorial artificial, capaz de provocar um conflito vestíbulo ocular, além da mudança do ganho desse mesmo reflexo (DI GIROLAMO et al., 2001).

A realidade virtual tem menor eficácia no paciente que apresenta comprometimento do sistema nervoso central: é o que apresenta pior resposta à terapia, ocorrendo em $50 \%$ dos casos (BITTAR et al., 2002). Ainda de acordo com referidos autores, em um levantamento de 155 pacientes, as síndromes de etiologia central foram a primeira causa de limitação da resposta ao tratamento com realidade virtual. Para a literatura especializada, a experiência mostrou que as lesões cerebelares - embora respondam à realidade virtual - são as que apresentam pior prognóstico entre as lesões centrais (BROWN et al., 2006). Apesar dos relatos, Brown et al.(2006) fazem a ressalva de que a realidade virtual é eventualmente a única terapia que surte algum efeito benéfico nos pa- cientes, muitas vezes acamados, dotados de lesões centrais. Embora não se espere resolução dos problemas do equilíbrio corporal, a realidade virtual melhora o desequilíbrio e é altamente benéfica para esses indivíduos. Santos et al.(2013) referem-se à realidade virtual como sendo, na atualidade, uma nova opção de tratamento que pode contribuir para a melhora do equilíbrio em pacientes neurológicos. Tal estratégia se diferencia do tratamento tradicional, sendo uma forma de estimular o indivíduo à adesão ao tratamento. Os autores ainda ressaltam que essa abordagem pode ser incentivada aos sequelados de alterações neurológicas, uma vez que as pesquisas existentes vêm demonstrando como favoráveis os benefícios desse recurso para a melhora do equilíbrio corporal.

Considerando a relevância do tema para a Fonoaudiologia - em especial, na área da Otoneurologia - assim como a conduta adotada e a evolução do paciente em questão, é que nos motivaram a relatar o presente caso, o qual visa a descrever a contribuição da terapia labiríntica baseada na habituação no paciente acometido por trauma crânio encefálico com sequelas cerebelar e labiríntica.

\section{DESCRIÇÃO DO CASO CLÍNICO}

Trata-se de um relato de caso clínico, com dados da avaliação e descrição dos procedimentos de avaliação e reabilitação do equilíbrio corporal. Este estudo de caso atende aos preceitos éticos pertinentes, por meio do parecer favorável sob o no 253.990/2013, emitido pelo Comitê de Ética em Pesquisa da Universidade do Estado da Bahia, seguido da assinatura do Termo de Consentimento Livre e Esclarecido.

O paciente D.G.S., sexo masculino, 55 anos, vem sendo acompanhado no Setor de Otoneurologia da Clínica Escola de Fonoaudiologia da Universidade do Estado da Bahia (UNEB), desde 2008 até outubro de 2013. O paciente em questão foi vítima de uma coronhada de arma de fogo em região da cabeça, tendo como consequência um traumatismo crânio encefálico. Foi encaminhado pelo Neurologista para o Programa de Reabilitação Vestibular na Clínica Escola de Fonoaudiologia da UNEB com seguinte laudo neurológico: "Paciente apresenta movimentos involuntários com síndrome labiríntica e cerebelar secundária a traumatismo crânio encefálico (TCE) grave. CID 10: H 83.2, S 09.2, H 91.3". Segundo impressão diagnóstica por ressonância magnética tinha a seguinte descrição: "Cóclea esquerda parcialmente individualizada, com área de moderada esclerose óssea em labirinto membranoso". Tais laudos foram emitidos pelo neurologista e radiologista, respectivamente. No laudo audiológico, emitido pelo fonoaudiólogo que avaliou o paciente, apresentou o seguinte resultado: “OD Limiares auditivos normais. OE - Ausência de resposta na máxima intensidade do audiômetro; curvas timpanométricas: tipo ' $\mathrm{A}$ ', com ausência de reflexos acústicos estapedianos contralaterais bilateralmente". Segundo pro- 
pedêutica otoneurológica de 2008 , o paciente relatou as seguintes queixas: tontura constante (tipo vertigens objetiva e subjetiva), desequilíbrio, distorção visual em situações de movimentos rápidos de cabeça, bem como durante a deambulação, ao olhar para cima, deitado e ao levantar-se rápido. Apresentava os seguintes sintomas associados: queda, formigamento nas extremidades, hipoacusia, cefaleia, náuseas, sensação de plenitude auricular, dor em região cervical. Referia, ainda, sentir zumbido intenso e constante no ouvido esquerdo de $p i-$ tch grave e agudo, mas tal sintoma não apresentava relação direta com a tontura. O paciente ainda não havia se submetido à avaliação do sistema vestibular devido ao seu quadro geral. Quanto aos hábitos deletérios, era tabagista e fazia uso de cafeína regularmente. Com relação aos medicamentos utilizados, fazia uso de depressor labiríntico, antidepressivos e ansiolíticos diariamente. 0 paciente em estudo utilizava uma muleta para conseguir se locomover. As imagens a seguir ilustram o estado de deambulação antes da intervenção, com e sem o auxílio de muletas.

\section{METODOLOGIA}

A intervenção fonoaudiológica teve como objetivo a remissão da tontura e o aumento da estabilidade durante a deambulação. O paciente ingressou no programa de reabilitação vestibular do Setor de Otoneurologia da Clínica Escola de Fonoaudiologia da UNEB, onde foram realizados os seguintes procedimentos:

1- Explanação acerca do funcionamento do programa;

2- Exposição teórica sobre anatomofisiopatologia do sistema vestibular;

3-Anamnese fonoaudiológica direcionada aos sistemas auditivo e vestibular;

4-Aplicação do DHI (Dizziness Handicap Inventory) pré e pós-tratamento;

5- Utilização de protocolo de relaxamento de cintura escapular;

6- Realização de estimulação optocinética;

7- Realização dos exercícios dos protocolos de Cawthorne e Cooksey, do Zee e da Associação Italiana de Otologia;

8- Utilização de jogos com realidade virtual - Knect XBox.

Figuras 1 e 2. Deambulação com muleta. Registro fotográfico do paciente em atividade terapêutica, 2012.
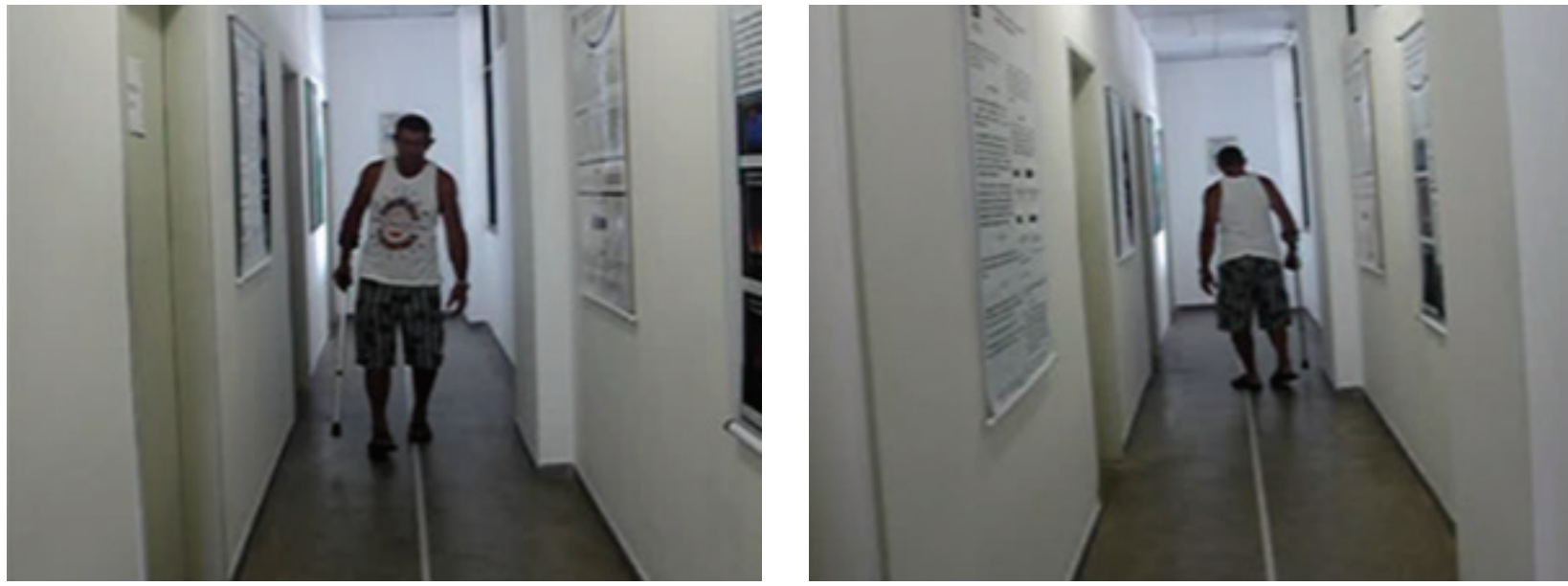

Figuras 3 e 4. Deambulação sem muleta. Registro fotográfico do paciente em atividade terapêutica, 2012.
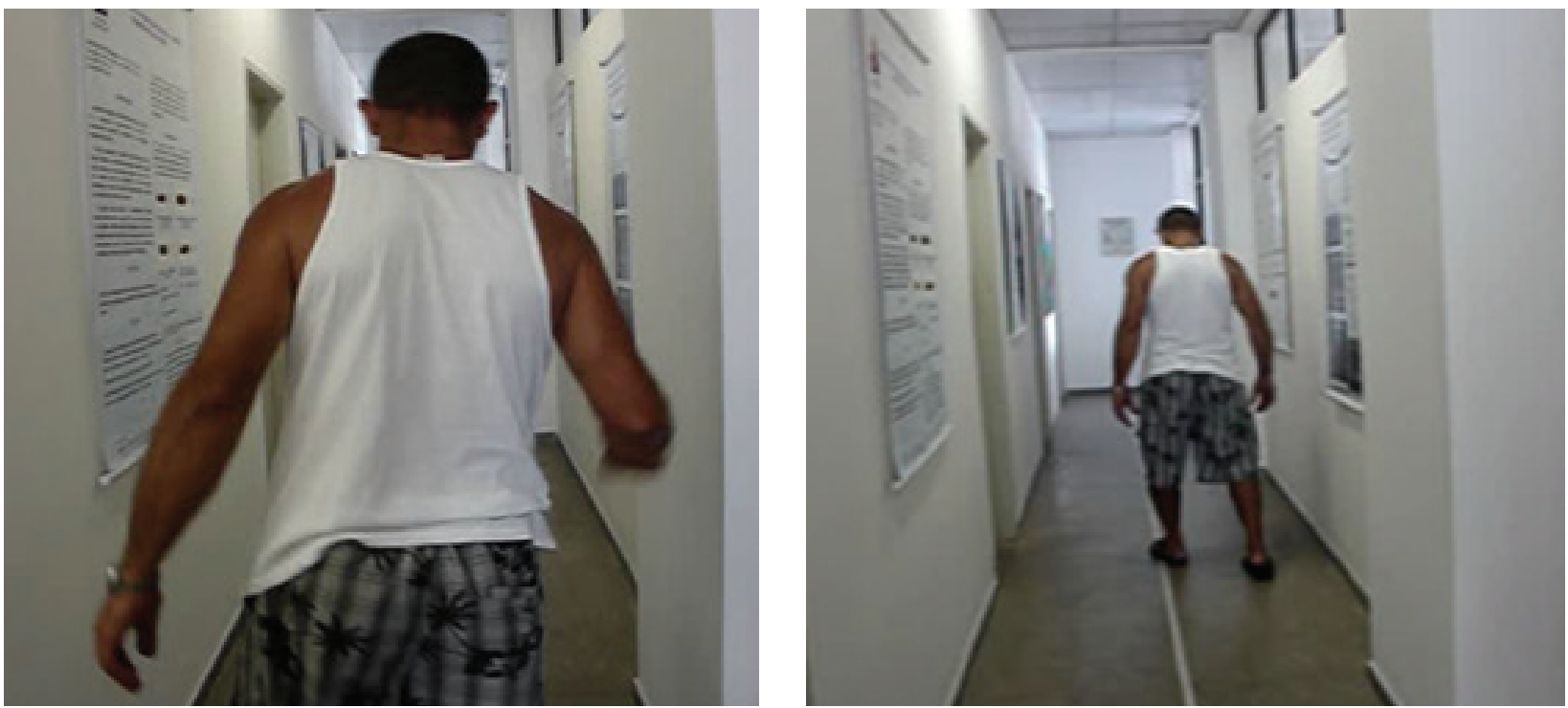


\section{RESULTADOS}

Baseado nos resultados da avaliação otoneurológica, em 2008, foi proposto exercícios do protocolo de Cawthorne e Cooksey (1940), realizados na íntegra, em conjunto ao trabalho do reflexo vestíbulo ocular (RVO) por meio da utilização do estimulador optocinético, modelo TB 103, da marca Berger. Antes do paciente em estudo iniciar o programa, foi realizado a aplicação do $\mathrm{DHI}$ pré tratamento, o qual apresentou um escore de 84 pontos. O paciente foi orientado a realizar, em casa, os exercícios propostos na terapia, em uma frequência de três vezes ao dia (dez vezes cada exercício), diariamente - acompanhados de registro semanal de sua evolução, acerca dos sintomas percebidos, quando do comparecimento ao serviço de reabilitação vestibular uma vez na semana.

Em 2009, foram propostos os exercícios do protocolo do Zee associados à estimulação optocinética, durante a sessão semanal.

Devido à evolução satisfatória quanto à recuperação do equilíbrio corporal do paciente, em 2010, optou-se por manter a mesma proposta terapêutica, preservando a metologia anterior.

Em 2011, o paciente foi submetido aos exercícios do Protocolo de Reabilitação Vestibular da Associação Italiana de Otologia. Naquela ocasião, o paciente em estudo já não mais utilizava muleta para se locomover. Segundo ele, já conseguia caminhar, ainda que com um pouco de dificuldade, o que já era um progresso visível, e que não iria mais utilizar a referida muleta. Naquele período, foi orientado que, ao sair sozinho, principalmente quando atravessasse ruas e avenidas, solicitasse ajuda de alguém.

Segundo propedêutica otoneurológica de 2012, o paciente relatou desequilíbrio, tontura esporádica, do tipo vertigem subjetiva, oscilopsia em situações de movimentos rápidos de cabeça, quando da deambulação e ao levantar-se rápido. O mesmo também fazia referência a sintomas como cefaleia, náuseas, sensação de plenitude auricular e dor em região cervical. Queixou-se de zumbido intenso e intermitente no ouvido esquerdo de pitch grave, o qual aumentava com a tontura. Naquela ocasião, fazia uso de medicamentos do tipo ansiolítico e antiemético. Foi possível realizar a avaliação da função labiríntica, uma vez que já havia sido trabalhado o sistema vestibular o que possibilitaria sua avaliação. Vale ressaltar que o paciente deixou de utilizar a muleta para se locomover, bem como conseguiu realizar a avaliação do sistema labiríntico, o qual demanda a supressão de um dos sistemas que envolvem o equilíbrio, o que confere um resultado satisfatório quanto à estimulação do labirinto contralateral com compensação vestibular central. O resultado da avaliação foi compatível com alteração vestibular periférica deficitária à esquerda, confirmando a lesão dos sistemas auditivo e vestibular esquerdo, não havendo respostas aos estímulos durante a avaliação do sistema labiríntico. Realizar a avaliação do sistema labiríntico representou, do ponto de vista funcional, uma constatação de que tal sistema foi efetivamente trabaIhado, ainda que com auxílio do labirinto contralateral, juntamente aos sistemas visual e proprioceptivo.
Em 2013, o paciente relatou desequilíbrio, tontura esporádica, do tipo vertigem subjetiva em situações de movimentos rápidos de cabeça e ao levantar-se de forma rápida. Quanto à deambulação, apresentou pouco desequilíbrio sendo o mesmo de forma assistemática e involuntária. Sintomas como cefaleia, náuseas, sensação de plenitude auricular diminuíram em intensidade e frequência, segundo relato. Além disso, queixou-se de zumbido intermitente no ouvido esquerdo de pitch grave sem relação com a tontura. O paciente em estudo ainda faz uso esporádico do tabaco e de medicamento do tipo anticonvulsivante, de acordo com prescrição médica.

A partir de maio de 2013 , o paciente se encontra em alta assistida quanto aos exercícios convencionais dos protocolos até então utilizados, porém iniciou-se a utilização de jogos com realidade virtual a fim de simular movimentos do dia a dia, proporcionando-lhe maior autonomia e segurança, implicando uma melhora na sua qualidade de vida. Na ocasião, o DHI apresentou um escore de 38 pontos. Durante sete meses, tal procedimento será realizado em sessões semanais, dentro do programa de reabilitação vestibular, com vistas à alta fonoaudiológica após esse período.

\section{DISCUSSÃO}

Os protocolos de reabilitação vestibular existentes para o tratamento da tontura são bastante variados, pois abrangem uma gama de possibilidades de estímulos nos sistemas proprioceptivo, visual e vestibular. No entanto, a escolha de determinados protocolos deve ser bastante cuidadosa, uma vez que o princípio é que a terapêutica deva ser personalizada no intuito de respeitar os limites de cada paciente (SOARES, 2006), bem como suas condições músculoesqueléticas na adequação dos exercícios, sendo de forma individualizada, conforme a situação do paciente (TEIXEIRA et al., 2010). O paciente em questão necessitou de cuidados específicos uma vez que corria risco iminente de queda durante a realização dos exercícios, fato este destacado pelos autores supracitados.

A literatura científica faz referência que, nos casos de lesão no SNC cujas estruturas que participam direta e indiretamente no processo de compensação vestibular central encontram-se comprometidas, uma vez que havendo a ausência de um sistema vestibular funcional, o SNC apresenta dificuldades para integrar de forma correta e adequada as informações advindas dos outros sistemas aferentes, visuais e proprioceptivos (BITTAR; BARROS, 2011). No entanto, um estudo recente mostra que, nos casos de ataxia, poucos pacientes relatam os efeitos das terapias de reabilitação, apesar de promover benefícios, ainda que não alcancem resultados definitivos nem uma melhora plena (MISSAOUI; THOUMIE, 2009). No presente estudo, o caso apresentado corrobora a opinião/relato deste último autor uma vez que se sabe da irreversibilidade das lesões do SNC; entretanto, a neuroplasticidade explica (dá o suporte científico) a melhora dos pacientes quando submetidos à terapia labiríntica quando o processo da habituação é alcançado. 
A definição dos procedimentos a serem adotados no tratamento do indivíduo portador de tontura secundária ao TCE é bastante eclética. De acordo com Teixeira et al. (2010) a reabilitação vestibular é efetiva em casos de doenças do SNC, principalmente quando os protocolos convencionais são associados a terapias alternativas em prol de uma meIhora das queixas. Missaoui e Thoumie (2009) reforçam a importância de os pacientes serem tratados em uma perspectiva multissensorial, ou seja, estimulando os sistemas visual, vestibular e proprioceptivo, de acordo com seu nível de deficiência, sendo a forma mais adequada para reduzir o comprometimento do equilíbrio em pacientes com esse tipo de lesão. Já Alsalaheen et al. (2013) consideram importante quando se faz a compreensão dos programas de reabilitação vestibular no tratamento de indivíduos pós-concussão, pois os padrões de prescrição de médicos especialistas podem elucidar as deficiências vestibulares, nesses casos, fornecendo aos terapeutas recursos cada vez melhores, no que concerne à qualidade do atendimento relacionada à prescrição de exercícios.

Segundo Lima et al. (2011), em estudo com pacientes portadores de ataxia cerebelar, a indicação do tratamento da reabilitação vestibular foi bem sucedida, uma vez que obtiveram melhora da função e equilíbrio ou estabilização do quadro neurológico desses pacientes.

No caso clínico ora em discussão, foi possível, com uma proposta de tratamento diversificada e inovadora, alcançar uma melhora efetiva na qualidade de vida dos pacientes quanto aos aspectos físico, emocional e funcional, o que vem a corroborar os estudos de Teixeira et al (2010). Tal resultado vem ao encontro do estudo de Barcala et al. (2011) o qual conclui, em seu relato, o benefício de exercício para o equilíbrio corporal e em prol de uma qualidade de vida, por meio do treino com jogos de realidade virtual - Wii Fit, considerando-o como mais um recurso terapêutico para os pacientes hemiparéticos. Schivinato et al. (2010) reforçam ainda que, além dos benefícios aos pacientes neurológicos por meio dos jogos de realidade virtual em seu estudo - o que vem a diferenciar de uma abordagem tradicional - deve-se ressaltar a importância desse tipo de estímulo e da adesão dos pacientes ao tratamento (SANTOS et al., 2013).

No presente estudo, a abordagem terapêutica instituída converge aos estudos realizados por Rodrigues et al. (2009) e Hoffer et al. (2004). Segundo Meli et al. (2006), a reabilitação vestibular proporciona uma melhor qualidade de vida aos pacientes, uma vez que reduz o grau de deficiência e melhora a capacidade de realizar tarefas diárias.

\section{CONCLUSÃO}

Com base nos resultados obtidos, pode-se afirmar que a utilização de protocolos diversos na composição de um programa que reabilite o sistema labiríntico, principalmente nos pacientes portadores de vestibulopatias mistas (como o caso ora exposto), favoreceu a recuperação das atividades básicas do dia a dia desse paciente.

Sendo a integridade do SNC primordial no processo de compensação vestibular central e, ao estar afetado de alguma forma, vale integrar/agregar aos protocolos tradicionais propostas terapêuticas que possibilitem atingir o fenômeno da habituação. Aderindo ao tratamento, sobretudo quando o paciente acredita na terapêutica instituída e persiste na sua prática, a recuperação do equilíbrio corporal e a autonomia do paciente tornam-se mais efetivas, o que se comprova nas imagens a seguir:

Figuras 5 e 6. Deambulação após intervenção. Registro fotográfico do paciente em atividade terapêutica, 2012.

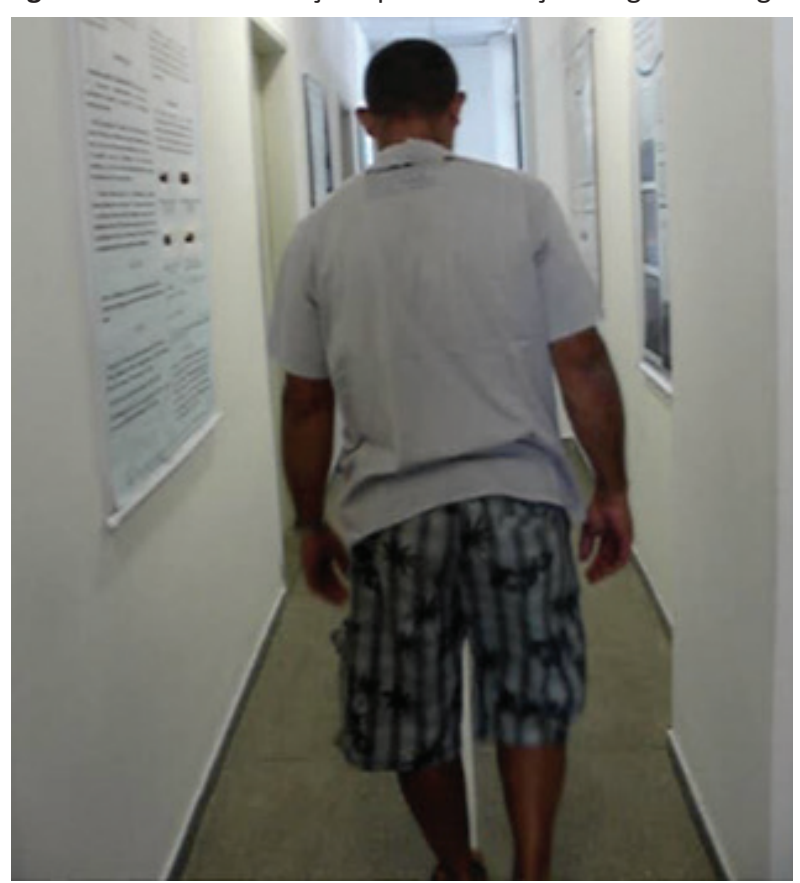




\section{REFERÊNCIAS}

1. ALSALAHEEN, B. A. et al. Exercise prescription patterns in patients treated with vestibular rehabilitation after concussion. Physiother. Res. Int., EUA, v.18, n. 2, p.100-108, 2013.

2. BARCALA, L. et al. Balance analysis in hemiparetics after training with wii fit program. Fisioter. Mov., Curitiba, v. 24, n. 2, p. 337-343, abr.- jun., 2011.

3. BARILLI, E. C. V. C. et al. A tecnologia de realidade virtual como recurso para formação em saúde pública à distância: uma aplicação para a aprendizagem dos procedimentos antropométricos. Ciênc. Saúde Coletiva, Rio de Janeiro, 2013. v. 16, supl. 11, p. 1227-1256.

4. BITTAR, R. S. M. et al. Síndrome do desequilíbrio no idoso. Pró-fono: Revista de Atualização Científica, Barueri, v.1, n.14, p.119-128, jan. abr. 2002.

5. BITTAR, R. S. M.; BARROS, C. G. C. Reabilitação vestibular por biofeedback no desequilíbrio corporal de origem central. Rev. Bras. Otorrinolaringol., São Paulo, v. 77, n.3, p.356-61, 2011.

6. BROWN, A. et al., A survey of very-long-term outcomes after traumatic brain injury among membrs of a population-based incident cohort. J. Neurotr., New Rochelle/ NY, v. 28, n. 2, p.167-176, 2011.

7. CABRAL, G. T. R. Interferência da queixa de tontura na qualidade de vida dos idosos cadastrados na UBS do bairro Araçás, Vila Velha/ES, Acta Otolaryngol./Técnicas em Otorrinolaringologia, Stokolm, v. 27, n.2, p.58-63, 2009.

8. DÍAZ, A. P. Transtornos psiquiátricos e qualidade de vida após traumatismo crânio-encefálico grave: um estudo prospectivo. 2011. $89 \mathrm{f}$. Dissertação (Mestrado em Ciências da Saúde) - Centro de Ciências da Saúde, Universidade Federal de Santa Catarina, Paraná, 2011.

9. DI GIROLAMO, S. Vestibulo-ocular reflex modification after virtual environment exposure. Acta Otolaryngol., London, v. 121, n.29, p. 211-5, 2001.

10. FONSECA, T. C. C. Avaliação e tratamento pós-hospitalar do traumatismo crânio-encefálico. 2013. 36 f. Tese (Doutorado em Medicina)

- Faculdade de Medicina, Universidade de Lisboa, Portugal, 2013.

11. GANANÇA, et al. Como diagnosticar e tratar vertigem. Rev. Bras. Med., São Paulo, v. 65, n.1, p.6-14, 2008.

12. GANANÇA, M. M.; CAOVILLA, H. H. Desequilíbrio e reequilíbrio. In: GANANÇA, M. M. Vertigem tem cura? São Paulo: Lemos, 1998; p. 1319.

13. GANANÇA, M. M.; CAOVILLA, H.H., MUNHOZ, M.S.L. Xeque-mate nas tonturas. Acta AWHO, São Paulo, v.16, n.3, p.129-37, 1997.

14. HOFFER, M. E. Characterizing and treating dizziness after mild head injury. Otol. Neurotol., Hagerstown, v. 25, n. 2, p.135-138, 2004.

15. HOFFER, M. E. et al. Vestibular consequences of mTBI. In: Traumatic Brain Injury: a Clinician's Guide to Diagnosis, Management, and Rehabilitation. p. 139-147, 2012. [S.I.], (Online) Disponível em: <http:// link.springer.com/chapter/10.1007/978-0-387-87887-4_8\#> Acesso em: 05 ago. 2013.
16. LIMA, N. et al. Efeitos da reabilitação vestibular na ataxia cerebelar: relato de três casos. Ensaios e Ciência, [S.I.], (editar no plugin de tradução o arquivo da citação ABNT), 15, out. 2011. Disponível em: <http:// sare.anhanguera.com/index.php/rensc/article/view/3058>. Acesso em: 07 Ago. 2013.

17. MASEL, B; DEWITT, D. Traumatic brain injury: a disease process, not an event. J. Neurotrauma, New York, v. 27, n. 8, p. 1529-1540, 2010.

18. MELI, A. et al. Vestibular rehabilitation and 6-month follow-up using objective and subjective measures. Acta Otolaryngol., London, v.126, n. 3, p. 259-266, 2006.

19. MISSAOUI, B.; THOUMIE, P. How far do patients with sensory ataxia benefit from so-called "proprioceptive rehabilitation"? Neurophysiol Clin., Paris, v. 39, n. 4-5, p. 229-33, 2009.

20. RECKARD, A. J. et al. Central vestibulopathy in a female collegiate basketball player: a case study. p. 105-10, 2013. Disponível em:

$<$ http://digitalcommons.fiu.edu/cgi/viewcontent.cgi?article $=1103 \&$ co ntext $=$ sferc \&sei-redir=1\&referer=http $\% 3 A \% 2 F \% 2 F s c h o l a r$.google. com.br\%2Fscholar\%3Fq\%3Drelated\%3Afov7tLrRFm8J\%3Ascholar. google.com\%2F\%26hl\%3Dpt-BR\%26as_sdt\%3D0\%2C5\#search=\%22rel ated\%3Afov7tLrRFm8J\%3Ascholar.google.com\%2F\%22> Acesso em: 07 ago. 2013.

21. RODRIGUES, et al. Vestibular rehabilitation with virtual reality in patients with Meniere's Disease. Rev. Equilíbrio Corporal e Saúde, São Paulo, v.1, p. 9-20, 2009.

22. SANTOS et al. Equilibrium in patients of brain injury who practice swimming and virtual reality. Rev. Neurocienc, São Paulo, v. 21, n.1, p. 89-93, 2013.

23. SCHIVINATO, A. M. et al. Influence of the wii fit on the balance of cerebellar dysfunction patient: case study. J Health Sci Inst., São Paulo, v. 28, n.1, p.50-2, 2010

24. SILVA, A. L. S.; MOREIRA, J. S. Vertigem: a abordagem da fisioterapia. Fisioterapia Brasil, São Paulo, v.1, n. 2, Nov.- Dez. 2000

25. SOARES, E. V. Reabilitação vestibular em idosos com desequilíbrios para marcha. Perspectivas, Campos dos Goytacazes, v. 6, n. 9, p. 88100,2006

26. SOCIEDADE BRASILEIRA DE OTORRINOLARINGOLOGIA. Consenso sobre vertigem. São Paulo: SOB, 2000. 38 p.

27. SHUMWAY-COOK, A. Reabilitação vestibular do paciente com traumatismo crânio encefálico. In: HERDMAN, S. J. (Coord.). Reabilitação vestibular. 2. ed. São Paulo: Manole, 2002. p. 472-486.

28. TAGUCHI, C.K. Reabilitação vestibular. In: DREUX, F.M.; MENDES, B.C.; NAVAS, A. L. (Org.). Tratado de fonoaudiologia da SBFa. São Paulo: Roca, 2009. p. 252-262.

29. TEIXEIRA, C. S. et al. Reabilitação vestibular: tendências e indicações. RBCEH, Passo Fundo, v. 7, n. 2, p. 280-288, maio/ago. 2010

Submetido em 13.11.2013;

Aceito em 20.12.2013. 